\title{
Caracterización geoquímica de las manifestaciones termales intermareales de Bahía Concepción en la Península de Baja California
}

\author{
María Luisa Leal-Acosta, Rosa María Prol-Ledesma
}

María Luisa Leal-Acosta

maluleal@angel.umar.mx

Instituto de Industrias, Universidad del Mar, Campus Puerto Ángel, Distrito de San Pedro Pochutla, Puerto Ángel, CP 70902, Oaxaca, México.

\section{Rosa María Prol-Ledesma}

Instituto de Geofisica, Universidad Nacional Autónoma de México, Ciudad Universitaria, Coyoacán, CP 045 10, CDMX, México.
BOL. SOC. GEOL. MEX. 2016

VOL. 68 NO. 3

P. $395-407$

Manuscrito recibido: Junio 19, 2014 Manuscrito corregido recibido: Agosto 17, 2015. Manuscrito aceptado: Septiembre 3, 2015.

\section{RESUMEN}

La presencia de manifestaciones hidrotermales en las costas de Bahía Concepción está asociada al régimen tectónico extensivo que generó la formación del Golfo de California. En este trabajo son analizadas las características geoquímicas de los manantiales intermareales para determinar la posible presencia de uno o varios yacimientos geotérmicos asociados con los diferentes grupos de manifestaciones superficiales. La temperatura de descarga más alta medida en los manantiales termales de la zona de estudio fue de $66{ }^{\circ} \mathrm{C}$, la cual se registró en la zona intermareal de $\mathrm{La}$ Posada. Las aguas termales de Bahía Concepción corresponden al tipo sódico-cloruradas y su composición química e isotópica indica la incidencia de fenómenos de mezcla con agua de mar y probablemente con agua de alta salinidad. Comparado con el agua del mar, el agua termal presentó bajas concentraciones de $\mathrm{Na}^{+}, \mathrm{Cl}^{-}, \mathrm{Mg}^{2+}, \mathrm{SO}_{4}{ }^{2-}$ y $\mathrm{HCO}_{3}^{-}$ $\mathrm{y}$ alta concentración de $\mathrm{Si}$ y cationes tales como $\mathrm{Ca}^{2+}, \mathrm{Sr}^{2+}$ y K $\mathrm{K}^{+}$. La composición isotópica indicó que el agua termal de las manifestaciones intermareales en la parte norte de la bahía, se ajustan a una línea de mezcla de agua de mar con un corrimiento hacia el enriquecimiento de ${ }^{18} \mathrm{O}$, debido a la interacción de agua con la roca. Por lo tanto, es probable que un solo reservorio alimente todos los manantiales analizados.

Palabras clave: geotermia, manantiales geotermales intermareales, yacimiento geotermal, México.

\section{ABSTRACT}

The presence of hydrothermal manifestations on the coast of Bahia Concepción is associated with an extensional tectonic regime related to the formation of the Gulf of California. In this work, the geochemical composition of the intertidal springs is analysed to characterize the geothermal reservoirs associated with different groups of superficial manifestations. The La Posada hot springs present the highest temperature of the intertidal springs in the study area $\left(66^{\circ} \mathrm{C}\right)$. The hot springs in Bahía Concepción correspond to the sodium-chloride type and its chemical and isotopic composition shows the incidence of mixing phenomena with seawater and probably with high salinity. Compared to seawater, the thermal water has lower concentrations of $\mathrm{Na}^{+}$, $\mathrm{Cl}, \mathrm{Mg}^{2+}, \mathrm{SO}_{4}{ }^{2+}$ and $\mathrm{HCO}_{3}^{-}$ions, and higher concentrations of $\mathrm{Si}$ and cations such as $\mathrm{Ca}^{2+}, \mathrm{Sr}^{2+}$ and $\mathrm{K}^{+}$. The isotopic composition indicates that the thermal water from the intertidal manifestations in the northern part of the bay lies along a mixing line of sea water with groundwater with a shift towards oxygen enrichment due to water-rock interaction. Therefore, it is likely that one single reservoir feeds all sampled hot springs.

Keywords: geothermics, intertidal geothermal hot springs, geothermal reservoir, Mexico. 


\section{Introducción}

La zona de Bahía Concepción presenta numerosas manifestaciones hidrotermales (Figura 1) que ya han sido estudiadas por diferentes autores (Prol-Ledesma et al., 2004; Canet et al., 2005a, b; Forrest et al., 2005; Villanueva-Estrada et al., 2012, 2013). La mayor parte de los estudios se han enfocado en las manifestaciones submarinas en la zona de Mapachitos y se han enfocado a identificar los procesos fisicoquímicos y bioquímicos que tienen lugar durante el ascenso y descarga de los fluidos hidrotermales; sin embargo, existen numerosos manantiales intermareales a lo largo de la costa oeste de la Bahía que no han sido analizados y de los cuales no se ha establecido la relación entre las diversas manifestaciones costeras y las ventilas submarinas.

Con base en los resultados de la modelación geoquímica, se ha propuesto que los fluidos termales son originados por la penetración de agua meteórica que es calentada a profundidad y posteriormente se mezcla con agua connata y previo a la descarga se mezcla con el agua del mar de la Bahía (Villanueva-Estrada et al., 2012). Este modelo se basa en las discrepancias con un modelo lineal de
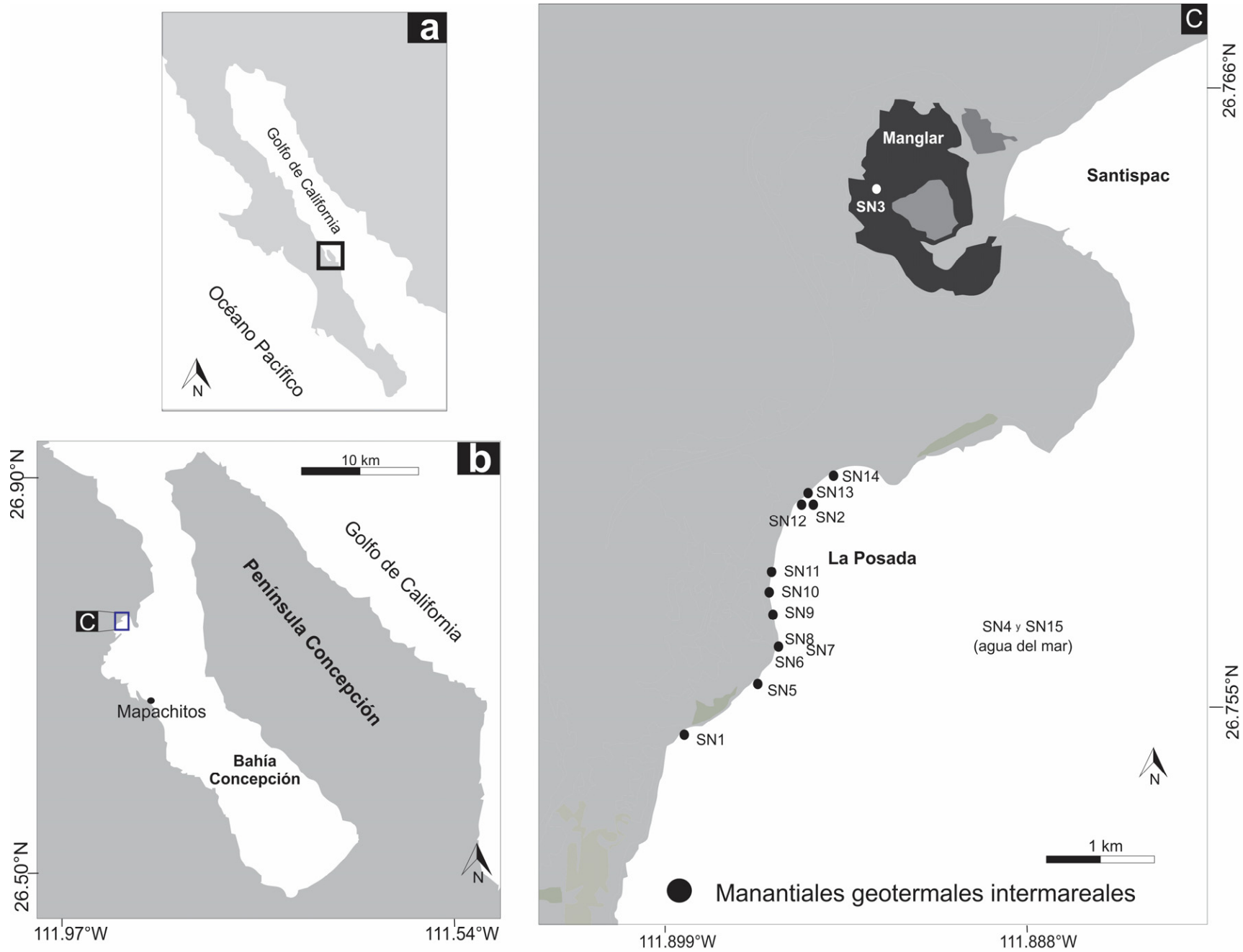

Figura 1 Mapas del área de estudio: a) Localización de la Bahía Concepción. b) Ubicación del área de estudio (Santispac y La Posada) y sitios de referencia. c) Sitios de muestreo de agua termal y agua de mar. 
mezcla entre el fluido geotérmico y el agua de mar para las manifestaciones intermareales, puesto que el agua termal presenta un enriquecimiento anómalo en $\mathrm{Ca}$ y $\mathrm{Cl}$ que puede deberse a la mezcla con aguas connatas. La composición química del agua termal muestra un enriquecimiento en $\mathrm{Ca}, \mathrm{As}, \mathrm{Hg}, \mathrm{Mn}, \mathrm{Ba}, \mathrm{HCO}_{3}, \mathrm{Li}, \mathrm{Sr}, \mathrm{B}, \mathrm{I}, \mathrm{Cs}, \mathrm{Fe}$ y Si con respecto al agua de mar (Prol-Ledesma et al., 2004). Esos autores determinaron la composición del miembro final termal (MFT) en las manifestaciones submarinas que concuerdan con un modelo lineal de mezcla con el agua de mar en la zona de Mapachitos, para posteriormente aplicar geotermómetros químicos para calcular la temperatura de equilibrio que tiene un valor de aproximadamente $200{ }^{\circ} \mathrm{C}$ (Prol-Ledesma et al., 2004), lo cual sería la temperatura indicada por la composición del agua termal previo a su mezcla con agua de mar.

Aproximadamente a $8 \mathrm{~km}$ al norte de la Ensenada Mapachitos, donde se localizan las ventilas submarinas, existen manantiales intermareales en el área de Santispac (Figura 1), donde los fluidos tienen temperaturas mayores a las del agua del mar circundante y bajo pH (Merchand-Reyes, 2010; Prol-Ledesma et al., 2004; Leal-Acosta et al., 2013). En los estudios previos se observó que las características químicas de los manantiales intermareales son diferentes de los de las ventilas submarinas localizadas al sur de la Bahía Concepción y por lo tanto deben seguir diferentes procesos de mezcla, por lo cual se plantea este trabajo para conocer la composición química e isotópica de las manifestaciones termales intermareales con el fin de dar información acerca de su composición y posible origen, qué sirva de antecedente en los estudios futuros sobre su potencial energético.

\section{Descripción del sitio de estudio}

\subsection{GARACTERÍSTICAS OCEANOGRÁFICAS Y GEOMORFOLÓGICAS}

La Bahía Concepción se ubica en la costa occidental del Golfo de California entre los $26^{\circ} 33^{\prime}$ y $26^{\circ} 53^{\prime}$ de latitud norte y $111^{\circ} 42^{\prime}$ y $112^{\circ} 56^{\prime}$ de longitud oeste (Figura 1). Posee una extensión de $275 \mathrm{~km}^{2}$, con $40 \mathrm{~km}$ de largo y $\sim 5$ a $10 \mathrm{~km}$ de ancho (Contreras, 1985). La bahía está orientada en los ejes NNW-SSE con la boca hacia el Golfo de California en la parte norte (Ledesma-Vázquez y Johnson, 2001). La comunicación con el Golfo de California consiste en un canal central con un umbral de $15 \mathrm{~m}$ de profundidad, orientado a la costa oeste. Las mayores profundidades son de 38 metros al sur de la bahía, en promedio en la parte central de la bahía la profundidad se mantiene en los $30 \mathrm{~m}$. Las mareas son de tipo semidiurnas con un rango de $59 \mathrm{~cm}$ en la entrada y hasta $75 \mathrm{~cm}$ en la cabeza (Obeso-Nieblas et al., 1996).

\subsection{GEOLOGÍA}

La revisión más reciente de la geología y tectónica de la zona de Bahía Concepción se realizó por Rodríguez-Díaz (2009), en la cual se enfatizan las características geológicas en relación con las manifestaciones hidrotermales. Las formaciones geológicas adyacentes a la Bahía Concepción pertenecen al Grupo Comondú. Están formados por conglomerados volcánicos intercalados, areniscas, brechas riolíticas y tobas, flujos de lava andesítica y lahares (Bustamante-García, 1999). Las rocas volcánicas del Grupo Comondú son principalmente calco-alcalinas y exhiben un amplio rango de $\mathrm{SiO}_{2}$ que corresponden a la composición de riolitas a basaltos (Biggiogero et al., 1995). Las unidades litológicas más recientes (Holoceno) consisten de sedimentos clásticos continentales y marinos, además de depósitos costeros, lacustres, aluviales y eólicos. También existen formaciones volcánicas que incluyen abundantes flujos basálticos y algunas grandes estructuras como las Tres Vírgenes y Volcán Mencenares (Camprubí et al., 2008). Algunas formaciones adyacentes a la Bahía Concepción son El Salto, Minitas, Pilares, Hornillos y Ricasón (McFall, 1968). Las rocas que predominan en estas formaciones son areniscas, brechas andesíticas, tobas y basaltos. En la formación Infierno existen secuencias carbonatadas y fósiles de un ambiente de manglar, algunas capas son nódulos de sílice 
amorfo. En el cerro Mencenares predominan las rocas fluidales riolíticas, pómez y obsidiana (Camprubí et al., 2008). En general la geoquímica de las rocas ígneas de la zona adyacente a la Bahía Concepción se controla por la existencia de brechas volcánicas, ignimbritas, andesitas basálticas y lavas, y en algunas partes tobas, dacitas y diques (Camprubí et al., 2008).

\section{Métodos}

Se realizaron dos muestreos en las manifestaciones intermareales de Bahía Concepción que se localizan en Santispac y La Posada (Figura 1b). En el primer muestreo en agosto de 2004, se colectó una muestra en La Posada (SN1), dos en la zona de Santispac (SN2 en la playa y SN3 en la zona de manglar), y una muestra de referencia de agua de mar colectada en la ensenada Santispac (SN4). En el segundo muestreo realizado en marzo del 2009, se colectaron 10 muestras en los manantiales intermareales del área de La Posada y una muestra de referencia de agua de mar en la zona adyacente lejana a los manantiales. En cada una de las estaciones se tomaron los parámetros fisicoquímicos de agua usando un multiparámetro HACH mo- delo sensION1 (Tabla 1). Los cationes $\mathrm{Ca}^{2+}, \mathrm{Li}^{+}$, $\mathrm{K}^{+}, \mathrm{Mg}_{2}^{+}$y $\mathrm{Na}^{+}$fueron determinados por cromatografía iónica, mientras los bicarbonatos fueron determinados en campo por volumetría (Merchand-Reyes, 2010). La validación del método se realizó usando el método de adición de estándares tanto a muestras de agua de mar, como a muestras de aguas hidrotermales. El porcentaje de recuperación/coeficiente de variación para cada uno de los cationes medidos se muestra en la Tabla 2. El análisis de bicarbonatos tuvo un $100.3 \%$ de recuperación y un coeficiente de variación de $1.3 \%$ (Merchand-Reyes, 2010). Los análisis de los aniones mayores como cloruros, bromuros y sulfatos se realizaron por cromatografía de líquidos del tipo iónico, con extracción de fase sólida como pretratamiento de la muestra (Ángeles-Razo, 2011). Para la validación del análisis químico se empleó el método de recuperación mediante la adición de estándares. Los porcentajes de recuperación y el coeficiente de variación se muestran en la Tabla 2 (Ángeles-Razo, 2011). Los elementos traza fueron determinados directamente en las muestras de agua usando un ICP/MS de alta resolución marca Finnigan Mat ELEMENT 2 en el laboratorio comercial Actlabs (Ancaster, Ontario) con una RDS $<1 \%$. Los límites de detección y coeficientes

Tabla 1. Localización de los sitios de muestreo y parámetros fisicoquímicos.

\begin{tabular}{|c|c|c|c|c|c|c|c|}
\hline Muestra & Latitud N & Longitud O & $\mathbf{T}^{\circ} \mathbf{C}$ & pH & S (UPS) & Muestreo & Tipo \\
\hline SN1 & $26^{\circ} 45^{\prime} 20^{\prime \prime}$ & $111^{\circ} 53^{\prime} 48^{\prime \prime}$ & 61 & 6.5 & 24 & \multirow{4}{*}{$1^{\mathrm{er}}$ muestreo } & \multirow{3}{*}{ Intermareal } \\
\hline $\mathrm{SN} 2$ & $26^{\circ} 45^{\prime} 30^{\prime \prime}$ & $111^{\circ} 53^{\prime} 41^{\prime \prime}$ & 66 & 6.2 & 27 & & \\
\hline SN3 & $26^{\circ} 45^{\prime} 45.04^{\prime \prime}$ & $111^{\circ} 51^{\prime} 37.02^{\prime \prime}$ & 40 & 7 & 27 & & \\
\hline SN4 & $26^{\circ} 41^{\prime} 2.5^{\prime \prime}$ & $111^{\circ} 51^{\prime} 30.1^{\prime \prime}$ & 30 & 8.1 & 33 & & Agua de mar \\
\hline SN5 & $26^{\circ} 45^{\prime} 21.92^{\prime \prime}$ & $111^{\circ} 53^{\prime} 43.23^{\prime \prime}$ & 49.2 & 7.3 & 21.8 & \multirow{11}{*}{$2^{\text {do }}$ muestreo } & \multirow{10}{*}{ Intermareal } \\
\hline SN6 & $26^{\circ} 45^{\prime} 23.43^{\prime \prime}$ & $111^{\circ} 53^{\prime} 42.48^{\prime \prime}$ & 56.1 & 7.7 & 21.8 & & \\
\hline SN7 & $26^{\circ} 45^{\prime} 23.43^{\prime \prime}$ & $111^{\circ} 53^{\prime} 42.48^{\prime \prime}$ & 56.4 & 6.8 & 23 & & \\
\hline SN8 & $26^{\circ} 45^{\prime} 23.43^{\prime \prime}$ & $111^{\circ} 53^{\prime} 42.48^{\prime \prime}$ & 52.3 & 6.3 & 18.5 & & \\
\hline SN9 & $26^{\circ} 45^{\prime} 24.56^{\prime \prime}$ & $111^{\circ} 53^{\prime} 42.37^{\prime \prime}$ & 55.7 & 6.8 & 22.1 & & \\
\hline SN10 & $26^{\circ} 45^{\prime} 25.8^{\prime \prime}$ & $111^{\circ} 53^{\prime} 42.48^{\prime \prime}$ & 50.9 & 7.2 & 22 & & \\
\hline SN11 & $26^{\circ} 45^{\prime} 26.61^{\prime \prime}$ & $111^{\circ} 53^{\prime} 42.91^{\prime \prime}$ & 50.1 & 6.8 & 23.2 & & \\
\hline SN12 & $26^{\circ} 45^{\prime} 29.6^{\prime \prime}$ & $111^{\circ} 53^{\prime} 41.74^{\prime \prime}$ & 40.6 & 7.3 & 21.8 & & \\
\hline SN13 & $26^{\circ} 45^{\prime} 30.43^{\prime \prime}$ & $111^{\circ} 53^{\prime} 41.36^{\prime \prime}$ & 57.4 & 7.4 & 22.9 & & \\
\hline SN14 & $26^{\circ} 45^{\prime} 31.68^{\prime \prime}$ & $111^{\circ} 53^{\prime} 39.8^{\prime \prime}$ & 42.5 & 7.3 & 24.4 & & \\
\hline SN15 & $26^{\circ} 43^{\prime} 25.97^{\prime \prime}$ & $111^{\circ} 53^{\prime} 8.18^{\prime \prime}$ & 19 & 8.3 & 36 & & Agua de mar \\
\hline
\end{tabular}


Tabla 2. Porcentaje de recuperación y coeficientes de variación de los iones analizados.

\begin{tabular}{|c|c|c|}
\hline Ion & \% recuperación & CV \\
\hline $\mathrm{Li}^{+}$ & 98.8 & 1.1 \\
\hline $\mathrm{Na}^{+}$ & 101.9 & 2 \\
\hline $\mathrm{K}^{+}$ & 102 & 1.6 \\
\hline $\mathrm{Ca}^{2+}$ & 100.9 & 0.6 \\
\hline $\mathrm{Cl}^{-}$ & 98.9 & 0.4 \\
\hline $\mathrm{Br}^{-}$ & 100 & 1 \\
\hline $\mathrm{SO}_{4}{ }^{2-}$ & 99.9 & 0.1 \\
\hline $\mathrm{HCO}_{3}^{-}$ & 100.3 & 0.6 \\
\hline
\end{tabular}

de variación $(\mathrm{CV})$ se muestran en la Tabla 3. La composición isotópica de las muestras de agua termal fue determinada con espectrometría de masas en el laboratorio de LUGIS de la UNAM (Laboratorio Universitario de Geoquímica Isotópica) y los detalles del método son mostrados en Prol-Ledesma et al. (2004).

\section{Resultados}

\subsection{PARÁMETROS FISICOQUÍMICOS}

Los manantiales termales intermareales tienen un rango de temperatura de 40 a $66^{\circ} \mathrm{C}$. El agua de la manifestación intermareal localizada en La Posada tiene la máxima temperatura, mientras que en la zona de manglar en Santispac se registraron las temperaturas más bajas de $40{ }^{\circ} \mathrm{C}$. El agua de mar (muestras de referencia) tiene temperaturas de 19 ${ }^{\circ} \mathrm{C}$ y $30^{\circ} \mathrm{C}$ y esta variación se debe a la época del año en que fueron colectadas. $\mathrm{El} \mathrm{pH}$ en las aguas termales intermareales, tanto en el primero como en el segundo muestreo, varía entre 6.2 a 7.0 y 6.3 a 7.7 respectivamente. La salinidad de las aguas se encuentra en el intervalo entre 24 a 33 ups para el primer muestreo y 18.5 a 36 ups para el segundo muestreo, siendo la zona de manglar la de mayor salinidad (Tabla 1).

\subsection{GEOQUÍMICA DE LOS MANANTIALES TERMALES INTERMAREALES}

El contenido de iones mayores en los fluidos de los manantiales termales intermareales se muestra en la Tabla 3. Las aguas son del tipo sódico-cloruradas como se observa en el diagrama de Piper (Figura 2). Los contenidos de Ca disuelto son mayores en las aguas termales de Santispac con rangos de 32.44 a $34.68 \mathrm{mmol} \cdot \mathrm{L}^{-1}$. En La Posada las concentraciones de Ca varían en 16 a 30.8 $\mathrm{mmol} \cdot \mathrm{L}^{-1}$, mientras que en la muestra de agua de mar de referencia (SN15), las concentraciones de

Tabla 3. Composición química de las aguas termales colectadas en Bahía Concepción (Concentraciones en $\mathrm{mmol} \cdot \mathrm{L}^{-1}$ )

\begin{tabular}{|c|c|c|c|c|c|c|c|c|}
\hline Muestra & $\mathrm{Ca}^{2+}$ & $\mathrm{Mg}^{2+}$ & $\mathbf{K}^{+}$ & $\mathrm{Na}^{+}$ & $\mathrm{Cl}^{-}$ & $\mathrm{SO}_{4}{ }^{2-}$ & $\mathrm{HCO}_{3}^{-}$ & Si \\
\hline SN1 & 34.7 & 8.5 & 14.8 & 249.8 & 424.1 & 5.4 & 2.5 & 2.4 \\
\hline SN2 & 30.2 & 18.5 & 14.7 & 298.1 & 497.4 & 6 & 1.5 & 1.8 \\
\hline SN3 & 32.4 & 16.7 & 15 & 303.3 & 497.4 & 5.1 & 1.4 & 1.6 \\
\hline SN4 & 10.5 & 51 & 15.2 & 465.1 & 624.1 & 26.6 & 2.4 & 0 \\
\hline SN5 & 30.8 & 13.1 & 9.2 & 320.5 & 331.3 & 6.3 & 1.3 & 2.1 \\
\hline SN7 & 24.8 & 16.3 & 8.2 & 299.5 & 318.9 & 7.1 & 1.4 & 1.7 \\
\hline SN8 & 30.6 & 18.4 & 8 & 349.7 & 482.4 & 10.3 & 1.2 & 1.6 \\
\hline SN9 & 26.4 & 14.6 & 8.5 & 299 & 356.2 & 7.1 & 1.4 & 2.2 \\
\hline SN10 & 16 & 8.1 & 4.7 & 174.2 & 239.5 & 4.8 & 1.6 & 2 \\
\hline SN11 & 31.2 & 19.8 & 10.5 & 366.9 & 494.5 & 10.5 & 1.8 & 1.8 \\
\hline SN12 & 32.4 & 13 & 10.3 & 328.8 & 454.3 & 7 & 1.4 & 2 \\
\hline SN13 & 29.9 & 16.7 & 10 & 336.9 & 473.9 & 9 & 1.3 & 1.9 \\
\hline SN14 & 29.2 & 22 & 10.3 & 356.1 & 515.9 & 12.3 & 1.7 & 1.9 \\
\hline SN15 & 12.4 & 60.7 & 9.8 & 531.8 & 591.1 & 29.4 & 3.2 & nd \\
\hline
\end{tabular}

Límites de detección en mmol · L-1: Ca (0.006), Mg (0.006), K (0.001), $\mathrm{Na}(0.01), \mathrm{Cl}(0.001), \mathrm{SO}_{4}(0.0009), \mathrm{HCO}(0.06), \mathrm{Si}(0.0003, \mathrm{CV}$ 1.5); nd: no detectado. 


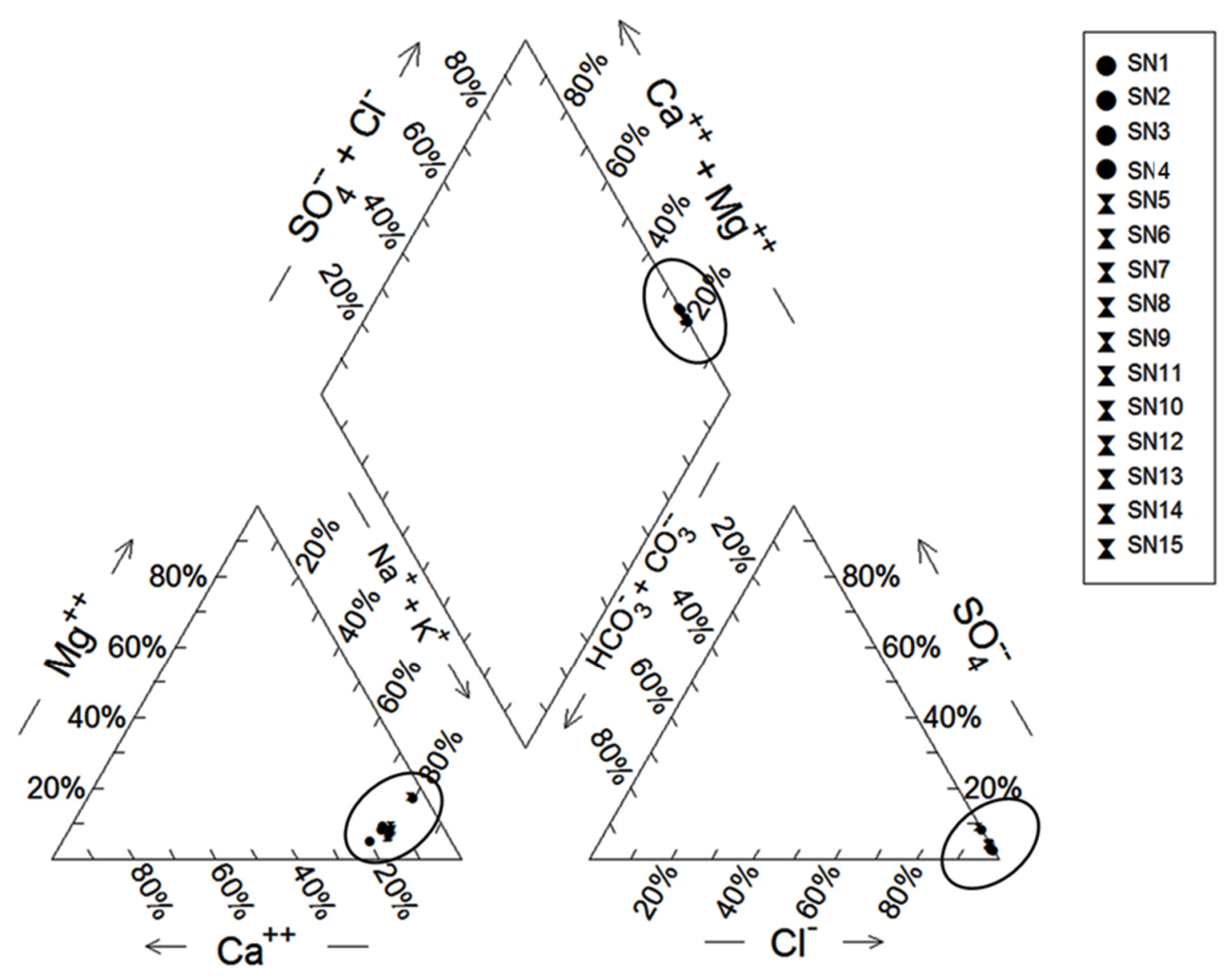

Figura 2 Diagrama de Piper que muestra la composición de los iones mayores de las aguas termales colectadas en Santispac y La Posada, Bahía Concepción: xprimer muestreo, • segundo muestreo

Ca tienen un menor intervalo de concentración que varió entre 10 y $12 \mathrm{mmol} \cdot \mathrm{L}^{-1}$ (Tabla 3 ). Las concentraciones de $\mathrm{K}$ variaron según la zona y en cada área son similares a lo detectado en la muestra de agua del mar de referencia, la cual tiene valores de $9.8 \mathrm{mmol} \cdot \mathrm{L}^{-1}$ (Tabla 3). En Santispac el contenido de $\mathrm{K}$ en las aguas hidrotermales tuvo un intervalo de 14.76 a $15.02 \mathrm{mmol} \cdot \mathrm{L}^{-1}$, mientras el agua del mar tiene $15.22 \mathrm{mmol} \cdot \mathrm{L}^{-1}$ (Tabla 3$)$. Las máximas concentraciones de $\mathrm{Mg}$ fueron de 18.48 $\mathrm{mmol} \cdot \mathrm{L}^{-1}$ en Santispac y $22.01 \mathrm{mmol} \cdot \mathrm{L}^{-1}$, que son bajos con respecto al agua de mar de referencia de las mismas zonas, con valores de $51.03 \mathrm{mmol} \cdot \mathrm{L}^{-1} \mathrm{y}$ $60.69 \mathrm{mmol} \cdot \mathrm{L}^{-1}$ respectivamente (Tabla 2). El sulfato tiene la misma tendencia con valores entre 5.1 y $6.4 \mathrm{mmol} \cdot \mathrm{L}^{-1}$ para La Posada y Santispac respectivamente, mientras el agua del mar de los sitios de referencia contiene de 26.6 a $29.4 \mathrm{mmol} \cdot \mathrm{L}^{-1}$, respectivamente. El Si es un indicador de aporte de aguas termales y los rangos de concentración variaron entre 1.56 a $2.42 \mathrm{mmol} \cdot \mathrm{L}^{-1}$ para todos los fluidos intermareales, mientras que el agua del mar presenta concentraciones 100 veces menores o por debajo del límite de detección (Tabla 3).

La concentración de elementos traza en los fluidos hidrotermales intermareales en Santispac y La Posada se muestran en la Tabla 4. La composición química de las aguas hidrotermales fue comparada separadamente para ambos muestreos respecto al agua de mar con respecto a las estaciones SN4 y SN15 para el primer y segundo muestreo respectivamente.

La composición de Mn, Ba, As, Mo, y W en los fluidos hidrotermales entre ambos muestreos no 
muestra diferencias significativas; sin embargo, se encuentran enriquecidos con respecto a la composición del agua del mar (con excepción de Mo). Las mayores concentraciones de B, Fe, Li y Sr se encontraron en los fluidos hidrotermales del primer muestreo (SN1-SN3) así como también las concentraciones determinadas en el agua de mar (SN4). Para estos mismos elementos, la composición de los fluidos hidrotermales del segundo muestreo es similar a la composición del agua del mar (Tabla 4). Tomando como elemento indicador de aporte de fluidos hidrotermales al Mn, este se encontró con altas concentraciones en las manifestaciones intermareales presentando intervalos de 19.8 a $50.9 \mu \mathrm{mol} \cdot \mathrm{L}^{-1}$. La mayor concentración de Mn fue medida en el manantial intermareal (SN2) localizado en La Posada $\left(50.9 \mu \mathrm{mol} \cdot \mathrm{L}^{-1}\right)$. Los contenidos de Ba también son bastante similares en ambas áreas con un intervalos de 0.4 a 2.5 $\mu \mathrm{mol} \cdot \mathrm{L}^{-1}$. Los elementos como B, Li y Sr presentan concentraciones significativamente mayores en La Posada (muestras SN1 a SN3) con intervalos altos de 290 a $899 \mu \mathrm{mol} \cdot \mathrm{L}^{-1}$ de B, 612 a $814 \mu \mathrm{mol} \cdot \mathrm{L}^{-1}$ de Li y 243 a $292 \mu \mathrm{mol} \cdot \mathrm{L}^{-1}$ de $\mathrm{Sr}$ (Tabla 4).

\subsection{COMPOSICIÓN ISOTOPICA}

La composición isotópica del agua termal se muestra en la Tabla 4. Las muestras de La Posada se encuentran dentro del intervalo de -3.9 a -2.6 para el $\delta^{18} \mathrm{O}_{\text {vSMOw }} \%$ y de -36 a -24 para $\delta \mathrm{D}_{\text {vSMOw }}$ $\%$, mientras las aguas termales colectadas en el primer muestreo (SN1 a SN3) oscilaron entre -2.7 a -3.9 para el $\delta^{18} \mathrm{O}_{\text {vsmow }} \%$ y -26.1 a -35.5 para el $\delta \mathrm{D}_{\text {vsmow }} \%$. Los datos de la composición isotópica de las aguas termales intermareales se encuentran por debajo de la línea de agua meteórica local y son congruentes con una mezcla de agua de mar cercana a Santispac con agua del miembro final termal calculado para Bahía Concepción y reportado por Prol-Ledesma et al., 2004 (Figura 3).

\section{Discusión}

\subsection{ORIGEN DE LAS MANIFESTACIONES TERMALES EN LA BAHÍA CONCEPCIÓN}

Las manifestaciones termales en Bahía Concepción están relacionadas con la actividad tectónica

Tabla 4. Concentración de elementos traza de las aguas termales intermareales colectadas en La Posada, Bahía Concepción (Concentraciones en $\mu \mathrm{mol} \cdot \mathrm{L}^{-1}$ ).

\begin{tabular}{|c|c|c|c|c|c|c|c|c|c|}
\hline Muestra & Mn & Ba & Br & B & As & Li & Fe & Sr & Mo \\
\hline SN1 & 28.8 & 2.5 & nd & 899 & 4.8 & 814 & 4.83 & 292 & 20 \\
\hline SN2 & 51 & 1.6 & nd & 763 & 3.6 & 583 & 19.87 & 243 & 20 \\
\hline SN3 & 2 & 0.4 & nd & 823 & 2 & 612 & 0.9 & 257 & 20 \\
\hline SN4 & nd & nd & nd & 290 & nd & nd & 0.36 & 78 & nd \\
\hline SN5 & 19.8 & 1.8 & 576 & 194 & 3.3 & 94 & 0.36 & 79 & 24 \\
\hline SN6 & 46.7 & 1.2 & nd & 185 & 4.1 & 87 & 0.62 & 79 & 24 \\
\hline SN7 & 36.6 & 0.9 & nd & 152 & 3.1 & 69 & 0.72 & 81 & 20 \\
\hline SN8 & 33.1 & 0.8 & 751 & 134 & 2.9 & 66 & 0.54 & 81 & 18 \\
\hline SN9 & 44.7 & 1.2 & 613 & 180 & 4.1 & 83 & 9.58 & 80 & 23 \\
\hline SN10 & 48.2 & 1.1 & 563 & 166 & 3.9 & 85 & 1.25 & 80 & 22 \\
\hline SN11 & 39.3 & 0.1 & 751 & 164 & 3.7 & 76 & nd & 80 & 23 \\
\hline SN12 & 34.1 & 1.3 & 701 & 180 & 4.7 & 95 & nd & 79 & 22 \\
\hline SN13 & 30.9 & 1.6 & 726 & 149 & 4.3 & 85 & nd & 80 & 23 \\
\hline SN14 & 0.2 & nd & 776 & 162 & 4.6 & 73 & nd & 80 & 23 \\
\hline SN15 & nd & nd & 663 & 101 & nd & nd & nd & 59 & 30 \\
\hline
\end{tabular}


asociada con fenómenos de extensión cortical y volcanismo en la península. Este fenómeno provoca un sistema de fallas en la costa este de la Península de Baja California que favorece la circulación profunda de agua meteórica. El paso del agua meteórica a través de las fallas provoca su calentamiento por el gradiente geotérmico o por la presencia de cuerpos intrusivos recientes. De acuerdo a McFall, 1968, la falla relacionada con la presencia de las ventilas hidrotermales someras y los manantiales geotermales en la zona de Santispac-La Posada-Mapachitos, se denomina falla El Requesón y tiene dirección NO-SE. Las manifestaciones hidrotermales se distribuyen a lo largo de $700 \mathrm{~m}$ del tramo de costa rocosa, predominando las rocas volcánicas andesíticas del Grupo Comondú. Las rocas están expuestas a lo largo de los acantilados y son controladas por un sistema de fallas que delinea el margen oeste de la bahía y actúan como un conducto para los fluidos hidrotermales (Forrest $e t$ al., 2005).

Además de las manifestaciones intermareales en Bahía Concepción, existen ventilas hidrotermales submarinas, provenientes del mismo sistema de fallas y son localizadas al sur de la Bahía en Mapachitos (Ledesma-Vázquez y Johnson, 2001). La composición química de los fluidos de las ventilas hidrotermales fueron reportadas previamente por Prol-Ledesma et al. (2004) y estos autores calcularon la composición química e isotópica del agua termal antes de los procesos de mezcla con agua de mar, el cual se muestra en la figura 3 . Al realizar la comparación entre la composición de elementos traza de los muestras de la zona intermareal con las muestras de las ventilas hidrotermales submarinas, se encontró que en la zona intermareal, las aguas termales tienen altas concentraciones de trazas, lo cual podría indicar que en la zona intermareal los fluidos están menos diluidos y mezclados con el agua del mar de la zona. De acuerdo a los modelos de mezcla usando $\mathrm{Mg}=0$ como indicador del miembro final termal para los fluidos provenientes de las ventilas submarinas son una mezcla de agua termal y agua del mar (Prol-Ledesma et al., 2004). Sin embargo, este mismo modelo de mez- cla no concuerda para las concentraciones de los manantiales termales intermareales, debido a que estos no muestran un comportamiento lineal, por lo que Villanueva-Estrada et al. (2012) proponen un modelo de mezcla entre tres miembros finales: agua termal-agua connata y agua de mar. Para identificar el origen se usó la composición isotópica, la Figura 3 muestra la relación isotópica entre las aguas hidrotermales intermareales, el agua del mar de ambos muestreos y el miembro final termal determinado previamente para el yacimiento por Prol-Ledesma et al. (2004) así como los resultados de ese primer muestreo para la única muestra de manantiales intermareales (muestra BC-10 de Prol-Ledesma et al., 2004). Se muestra una tendencia lineal, por lo que el agua termal de los manantiales intermareales se comporta como una mezcla del miembro final termal con el agua de mar de Santispac (Figura 3). Prol-Ledesma et al., 2004 mostraron diferencias en la composición isotópica del agua termal intermareal de la Bahía Concepción con el agua submarina de la misma zona, encontrando que el agua termal intermareal tiene una mezcla lineal con el miembro final termal (Figura 9, Prol-Ledesma et al., 2004). Los cálculos de temperatura usando geotermometría reportados por Prol-Ledesma et al., 2004 indicaron que el miembro final termal tendría una temperatura aproximada de $200^{\circ} \mathrm{C}$ usando geotermómetros de $\mathrm{T}(\mathrm{Na}-\mathrm{K}-\mathrm{Ca}$ ) y $\mathrm{T}(\mathrm{Si})$. Mientras las manifestaciones intermareales de Santispac se encuentran en equilibrio parcial y la temperatura calculada con el geotermómetro $\mathrm{Na} / \mathrm{K}$ (Giggenbach, 1988) varía entre 166 y $233{ }^{\circ} \mathrm{C}$ (Prol-Ledesma et al., 2004). En este trabajo, sin embargo, no se realizaron cálculos de temperatura usando geotermómetros debido a que las muestras se encuentran en el borde del equilibrio parcial.

\subsection{COMPARACIÓN DE LOS FLUIDOS INTERMAREALES CON LAS MANIFESTACIONES SUBMARINAS EN MAPACHITOS}

El tipo de agua encontrado en las manifestaciones hidrotermales submarinas e intermareales es sódico-clorurado (Prol-Ledesma et al., 2004). Sin 


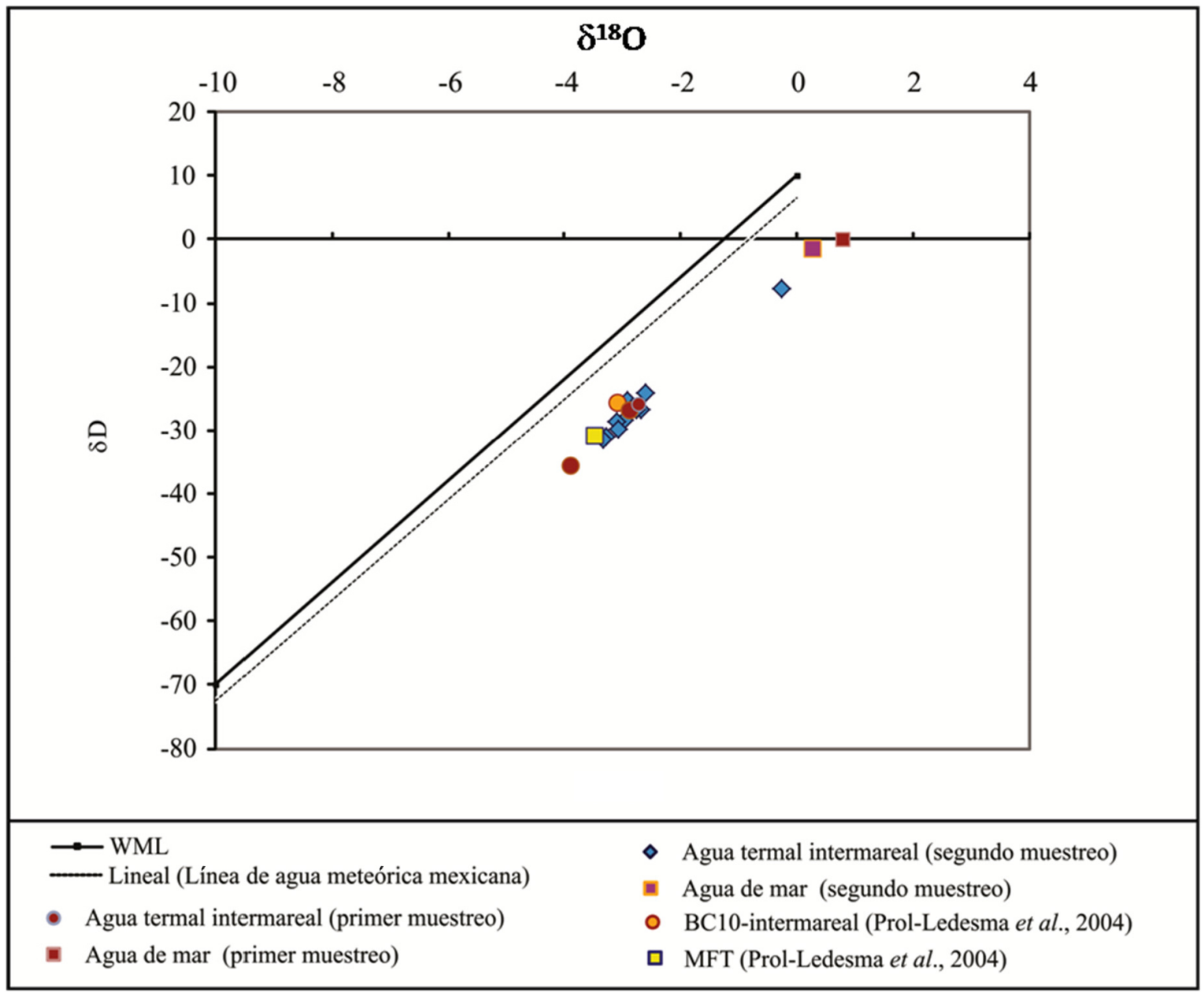

Figura 3 Comparación de la composición isotópica de las muestras termales intermareales colectadas en Bahía Concepción, del agua del mar (punto de control), agua del miembro final termal (MFT) del reservorio y agua intermareal BC10 reportados en Prol-Ledesma et al., 2004 que muestra una tendencia paralela con la World Meteoric Line (WML) y la línea de agua meteórica mexicana.

embargo, al comparar la composición química de las aguas termales intermareales con las muestras termales submarinas se observan diferencias en cuanto a la composición de iones mayores del agua del mar, ya que las aguas de las ventilas submarinas tienen las mayores concentraciones de $\mathrm{Na}, \mathrm{Cl}, \mathrm{Mg}, \mathrm{SO}_{4}, \mathrm{HCO}_{3}$ (Prol-Ledesma et al., 2004). Los manantiales costeros e intermareales presentan una alta concentración de Ca, mayor a $30 \mathrm{mmol} \cdot \mathrm{L}^{-1}$ y superior al Ca disuelto en las ventilas submarinas de Mapachitos y al agua de mar de referencia; por lo que el enriquecimiento de $\mathrm{Ca}$ en el agua de los manantiales intermareales y las ventilas hidrotermales sugiere que este elemento no es una contribución del agua de mar, sino que es un componente de un tercer miembro final (Villanueva-Estrada et al., 2012). Es importante señalar que en el caso de los manantiales intermareales, no se observó ninguna tendencia que indique un modelo de mezcla o de evaporación, excepto en las composiciones isotópicas del agua termal. Las concentraciones de $\mathrm{Mg}$ y $\mathrm{SO}_{4}$ en las zonas intermareales tienen valores menores a los de las ventilas submarinas, probablemente porque presentan una menor mezcla con agua de mar. Las bajas concentraciones de $\mathrm{Mg}$ medidas en las aguas hidrotermales de ambos ambientes (Santispac y Mapachitos) son las esperadas en el agua termal, ya que se ha reportado que, al calentar agua de mar, este elemento se precipita rápidamente en 
ciertos minerales que contienen este catión por lo que el agua caliente queda empobrecida en $\mathrm{Mg}$ (Bischoff y Seyfried, 1978).

En cuanto a los contenidos de Fe disuelto, este elemento tiende a formar precipitados en condiciones óxicas y en zonas hidrotermales tiende a depositarse cerca de su fuente dependiendo de los cambios de $\mathrm{pH}$ y la mezcla, lo cual es congruente con los minerales reportados por Canet et al. (2005a). Las aguas hidrotermales intermareales tienen baja concentración de Fe con intervalos de 0.36 a 4.83 $\mu \mathrm{mol} \cdot \mathrm{L}^{-1}$, esto puede ser debido a que se precipitan oxi-hidróxidos de Fe en la zona intermareal en Santispac y en la zona submarina de Mapachitos, Bahía Concepción (Canet et al., 2005b).

En Bahía Concepción no se observó diferencia entre los contenidos de Si en las aguas intermareales y las submarinas. Los fluidos de las ventilas tienen mayores contenidos de $\mathrm{Mn}, \mathrm{Br}$, As y Fe, al comparar con las intermareales. Esto puede ser porque los fluidos son más ricos en estos elementos o las condiciones intermareales favorecen la precipitación de los fluidos en las paredes de los manantiales intermareales. El manantial intermareal localizado en La Posada tiene una alta concentración de $\mathrm{Mn}\left(50.9 \mathrm{\mu mol} \cdot \mathrm{L}^{-1}\right)$, similar a lo reportado en las ventilas submarinas de Mapachitos (52.1 a $\left.64.8 \mu \mathrm{mol} \cdot \mathrm{L}^{-1}\right)$ (Prol-Ledesma et al., 2004).

En cuanto al aporte de elementos potencialmente tóxicos como As, en La Posada y Santispac las concentraciones de As disuelto se consideran bajas en ambos muestreos (2.9 a $4.7 \mu \mathrm{mol} \cdot \mathrm{L}^{-1}$ y 2 a 4.8 $\mu \mathrm{mol} \cdot \mathrm{L}^{-1}$ respectivamente) (Tabla 3 ). Las altas concentraciones de este elemento en Bahía Concepción han sido reportadas en la zona de las ventilas submarinas en Mapachitos con valores de 6.1 a $10.4 \mu \mathrm{mol} \cdot \mathrm{L}^{-1}$ (Prol-Ledesma et al., 2004) y estas altas concentraciones afectan a algunas especies. Leal-Acosta et al. (2013) reportaron los efectos de este elemento en las macroalgas marinas Sargassum sinicola de Santispac y Mapachitos; las macroalgas tuvieron contenidos de hasta $600 \mathrm{mg} \cdot \mathrm{kg}^{-1}$ en las muestras colectadas cerca de la fuente submarina. En particular este elemento ha sido estudiado con detalle y se demostró que se encuentra principal- mente adsorbido en los óxidos e hidróxidos de $\mathrm{Fe}$ (Villanueva-Estrada et al., 2013).

La composición isotópica de las aguas termales intermareales muestra diferencias importantes con las de las ventilas submarinas, ya que éstas tienen una composición isotópica comparable al agua meteórica local con rango de -0.3 a -3.1 para $\delta^{18} \mathrm{O}$ vsmow $\%$ y -0.3 a -25.5 para $\delta D_{\text {vsmow }} \%$ (Prol-Ledesma et al., 2004). Los manantiales intermareales tienen una composición isotópica que muestra un enriquecimiento en $\delta^{18} \mathrm{O}$ además de la mezcla entre el agua de mar (Tabla 5) con agua del miembro final termal reportado para esta misma zona por Prol-Ledesma et al. (2004).

\subsection{INFLUENCIA DE LAS AGUAS TERMALES DE BAHÍA CONCEPCIÓN SOBRE LA GEOQUÍMICA DEL ÁREA ADYACENTE A LAS FUENTES DE DESCARGA}

Ya que los fluidos son ricos en elementos mayores y traza, la precipitación de estos componentes es evidente, especialmente en la zona intermareal. Canet et al., 2005b caracterizaron la composición mineralógica de los precipitados acumulados en la zona de descarga de los manantiales. Estos corresponden a cortezas de óxido de manganeso y depósitos detríticos cementados por ópalo-A, barita y calcita, lo cual concuerda con la modelación química realizada para obtener los índices de saturación (Canet et al., 2005b). Las costras de óxido de $\mathrm{Mn}$ son ricas en $\mathrm{Ba}$ principalmente romanechita $\left[\left(\mathrm{Ba}, \mathrm{H}_{2} \mathrm{O}\right)_{2}\left(\mathrm{Mn}_{4}^{+}, \mathrm{Mn}_{3}^{+}\right)_{5} \mathrm{O}_{10}\right]$ y todorokita (Na, Ca, K) $\left(\mathrm{Mn}_{2}{ }^{+}, \mathrm{Mg}\right)\left(\mathrm{Mn}_{4}{ }^{+} \mathrm{O}_{12} \mathrm{xH}_{2} 0\right)$ ambas pobremente cristalizadas. Un estudio sobre la composición de elementos traza en costras de Mn colectadas en la zona intermareal de Santispac, mostraron altos contenidos de elementos como As $\left(635 \mathrm{mg} \cdot \mathrm{kg}^{-1}\right), \mathrm{Ba}\left(840 \mathrm{mg} \cdot \mathrm{kg}^{-1}\right), \mathrm{Hg}\left(60.3 \mathrm{mg} \cdot \mathrm{kg}^{-1}\right)$, y Mn $(10.35 \%)$ (Leal-Acosta et al., 2010). La fracción disuelta de elementos potencialmente tóxicos como As y $\mathrm{Hg}$ muestran concentraciones altas en los fluidos hidrotermales intermareales con respecto al agua del mar de Bahía Concepción con máximas de $109 \mu \mathrm{g} \cdot \mathrm{L}^{-1}$ y $45.3 \mu \mathrm{g} \cdot \mathrm{L}^{-1}$ para $\mathrm{As}$ y $\mathrm{Hg}$ respectivamente. Las máximas concentraciones para el agua del mar local son de $8.4 \mu g \cdot \mathrm{L}^{-1}$ y 21.3 
Tabla 5. Composición isotópica de oxígeno e hidrógeno en las muestras de aguas geotermales intermareales

\begin{tabular}{|c|c|c|}
\hline Muestra & $\boldsymbol{\delta}^{\mathbf{2}} \mathbf{H}_{\text {VSMOw }} \mathbf{\%}$ & $\boldsymbol{\delta}^{\mathbf{1 8}} \mathbf{O}_{\text {VSMOw }} \mathbf{~}$ \\
\hline SN1 & -36 & -3.9 \\
\hline SN2 & -27 & -2.9 \\
\hline SN3 & -26 & -2.7 \\
\hline SN4 & 0 & 0.8 \\
\hline SN5 & -31 & -3.3 \\
\hline SN6 & -25 & -2.9 \\
\hline SN7 & -27 & -2.9 \\
\hline SN8 & -28 & -3 \\
\hline SN9 & -29 & -3.1 \\
\hline SN10 & -27 & -2.7 \\
\hline SN11 & -32 & -3.3 \\
\hline SN12 & -27 & -2.8 \\
\hline SN13 & -24 & -2.6 \\
\hline SN14 & -30 & -3.1 \\
\hline SN15 & -1 & 0.3 \\
\hline
\end{tabular}

$\mu \mathrm{g} \cdot \mathrm{L}^{-1}$ para As y $\mathrm{Hg}$ respectivamente (Leal-Acosta et al., 2013). El impacto del aporte de elementos potencialmente tóxicos por fuentes hidrotermales en la Bahía Concepción se ha caracterizado con el uso de bioindicadores (macroalgas). Datos no publicados, obtenidos por Leal Acosta en 2012, muestran que las fuentes intermareales son fuente principal de Hg medido en Sargassum sinicola de Santispac con concentraciones de hasta $95 \mu \mathrm{g} \cdot \mathrm{kg}^{-1}$ de $\mathrm{Hg}$. Con respecto al $\mathrm{Hg}$, no se cuenta con evidencia de su acumulación en las macroalgas cercanas a las ventilas submarinas.

\section{Conclusiones}

Las aguas termales de las manifestaciones intermareales de Santispac y La Posada en Bahía Concepción corresponden a aguas del tipo sódico-cloruradas, con temperaturas de hasta $66^{\circ} \mathrm{C}$. $\mathrm{El}$ agua hidrotermal está enriquecida en elementos traza como Ca, Mn, Ba, Br, B, As, Li y Fe y la composición de elementos mayores en las aguas terma- les revela un empobrecimiento en $\mathrm{Na}, \mathrm{Cl}, \mathrm{SO}_{4}, \mathrm{y}$ $\mathrm{Mg}$ con respecto al agua de mar.

De acuerdo a la composición isotópica, el origen de los fluidos es una mezcla de agua del mar con el miembro final termal antes reportado para la misma zona, por lo que se infiere que todas las manifestaciones costeras y submarinas en la costa oeste de Bahía Concepción pueden tener un yacimiento común, lo cual implica un potencial geotérmico importante para esta zona que debe ser estudiado con medios geofísicos para determinar la extensión del yacimiento.

\section{Agradecimientos}

El trabajo contó con el apoyo del proyecto SENER-CONACyT Fondo de Sustentabilidad 152823 "Evaluación de los recursos geotérmicos de la Península de Baja California: continentales, costeros y submarinos" y UNAM-IMPULSA IV "Desalación de agua de mar con Energías Renovables". La autora principal (Leal-Acosta, M.L.) recibió un apoyo postdoctoral de CONACyT. Los autores agradecen el apoyo en el muestreo y el laboratorio de R.E. Villanueva, D. Rodríguez, J. Rentería.

\section{Referencias}

Ángeles-Razo, J., 2011, Determinación química de aniones en descargas naturales de la zona costera de Bahía Concepción, Baja California Sur, México: Cromatografía iónica: Mexico, D.F., Universidad Nacional Autónoma de México, Facultad de Química, Tesis de Licenciatura, 96 p.

Biggiogero, B., Chiesas, S., Zanchi, A. Montrasio, A., Vezzoli, L., 1995, The Cerro Mencenares Volcanic Center, Baja California Sur: source and tectonic control on postsubduction magmatism within the Gulf Rift: Geological Society of America Bulletin, 107, 1108-22. 
Bischoff, L.B., Seyfried, W.E., 1978,

Hydrothermal chemistry of seawater from $25^{\circ}$ to $350^{\circ} \mathrm{C}$ : American Journal of Science, 278, 838-860.

Bustamante-García, J., 1999, Monografía geológico-minera del estado de Baja California Sur: Pachuca, Hidalgo: Consejo de Recursos Minerales-Secretaría de Comercio y Fomento Industrial, 237 p.

Camprubí, A., Canet, C., Rodríguez-Díaz, A., Prol-Ledesma, R.M., Blanco-Florido, D., Villanueva, R.E., López-Sánchez, A., 2008, Geology, ore deposits and hydrothermal venting in Bahía Concepción, Baja California Sur, Mexico: Island Arc, 17, 6-25.

Canet, C., Prol-Ledesma, R.M., Torres-Alvarado, I., Gilg, H.A., Villanueva, R.E., Lozano, R., 2005a, Silica-carbonate stromatolites related to coastal hydrothermal venting in Bahía Concepción, Baja California Sur, Mexico: Sedimentary Geology, 174, 97-113.

Canet, C., Prol-Ledesma, R.M., Proenza, J., Rubio-Ramos, M. A., Forrest, M., TorresVera, M.A., Rodríguez-Díaz, A.A., 2005b, $\mathrm{Mn}-\mathrm{Ba}-\mathrm{Hg}$ mineralization at shallow submarine hydrothermal vents in Bahía Concepción, Baja California Sur, Mexico: Chemical Geology, 224, 96-112.

Contreras, F., 1985, Las lagunas costeras mexicanas: México, D.F., Ed. Secretaría de Pesca, Segunda edición, 263 p.

Forrest, M.J., Ledesma-Vázquez, J., Ussler III, W., Kulongoski, J. T., Hilton D.R., Greene, H.G., 2005, Gas geochemistry of a shallow submarine hydrothermal vent associated with El Requesón fault zone in Bahía Concepción, Baja California Sur, México: Chemical Geology, 224, 82-95.

Giggenbach, W.F., 1988, Geothermal solute equilibria. Derivation of Na-K-MgCa geoindicators: Geochimica et Cosmochimica Acta, 52, 2749-2765.
Leal-Acosta M.L., Shumilin, E., Mirlean, N., Sapozhnikov, D., Gordeev, V., 2010, Arsenic and Mercury Contamination of Sediments of Geothermal Springs, Mangrove Lagoon and the Santispac Bight, Bahía, Concepción, Baja California Peninsula: Bulletin of Environment Contamination and Toxicology, 85, 609-613.

Leal-Acosta, M.L., Shumilin, E., Mirlean, N., Delgadillo-Hinojosa, F., SánchezRodríguez, I., 2013, The impact of marine shallow-water hydrothermal venting on arsenic and mercury accumulation by seaweeds Sargassum sinicola in Concepcion Bay, Gulf of California:Environmental Science: Processes \& Impacts, 15, 470-477. Ledesma-Vázquez, J., Johnson, M.E., 2001,

Miocene-Pleistocene tectono-sedimentary evolution of Bahía Concepción region, Baja California Sur (México): Sedimentary Geology, 144, 83-96.

McFall, G.C., 1968, Reconnaissance geology of Concepcion Bay Area, Baja California, Mexico: Stanford University Publications in Geological Science, 10, 1-25.

Merchand-Reyes, G., 2010, Implementación de una metodología para el estudio químico de cationes y bicarbonato en manantiales de Bahía Concepción (Baja California Sur, México): México, D.F., Universidad Nacional Autónoma de México, Facultad de Química, Tesis de Licenciatura, 144 p.

Obeso-Nieblas, M., Alatorre-Mendieta, M.A., Jiménez-Illescas, A.R., 1996, Modelación de la marea en Bahía Concepción, B.C.S. México: Oceánides, 11, 1-8.

Prol-Ledesma, R.M., Canet, C., Torres-Vera, M.A., Forrest, M.J., Armienta, M.A., 2004, Vent fluid chemistry in Bahía Concepción coastal submarine hydrothermal system, Baja California Sur, México: Journal of Volcanology and Geothermal Research, 137, 311-328. 
Rodríguez-Díaz, A.A., 2009, Metalogenia del área mineralizada en manganeso de Bahía Concepción, Baja California: México, D.F., Universidad Nacional Autónoma de México, Instituto de Geofísica, Tesis de Maestría, $208 \mathrm{p}$.

Villanueva-Estrada, R.E., Prol-Ledesma, R.M., Rodríguez-Díaz, A. A., Canet, C., TorresAlvarado, I.S., González-Partida, E., 2012, Geochemical processes in an active shallow submarine hydrothermal system, Bahía Concepción, México: mixing or boiling?: International Geology Reviews, 54 (8), 907-919.

Villanueva-Estrada, R.E, Prol-Ledesma, R. M., Rodríguez-Díaz, A.A., Canet, C., Armienta, M.A., 2013, Arsenic in hot springs of Bahía Concepción, Baja California Peninsula, México: Chemical Geology, 348, 27-36. 